\title{
Erratum: Superstatistics and quantum entanglement in the isotropic spin-1/2 $X X$ dimer from a nonadditive thermodynamics perspective [Phys. Rev. E 104, 024139 (2021)]
}

\author{
Jorge David Castaño-Yepes $₫$ and Cristian Felipe Ramirez-Gutierrez
}

(Received 7 September 2021; published 27 September 2021)

DOI: 10.1103/PhysRevE.104.039903

The computation of Eqs. (16) is wrong. The mistake is derived from Eq. (14) where it was stated that $Z_{1}(\beta)=\mathcal{Z}\left(\beta^{\star}\right)$. The latter equation is not correct [i.e., $Z_{1}(\beta) \neq \mathcal{Z}\left(\beta^{\star}\right)$ ]. To eliminate nonphysical temperatures, it must be corrected.

From the identity $\hat{\rho}(\beta)=\hat{\varrho}\left(\beta^{*}\right)$ and from Eqs. (7) and (12), Eq. (16b) is given by

$$
U=\frac{\operatorname{Tr}\left[\hat{\varrho}^{q}\left(\beta^{\star}\right) \hat{H}\right]}{\operatorname{Tr}\left[\hat{\varrho}^{q}\left(\beta^{\star}\right)\right]}=\frac{\mathcal{U}}{\operatorname{Tr}\left[\varrho^{q}\left(\beta^{\star}\right)\right]}=-\frac{\partial_{\beta^{\star}} \ln _{q} \mathcal{Z}}{\operatorname{Tr}\left[\hat{\varrho}^{q}\left(\beta^{\star}\right)\right]} .
$$
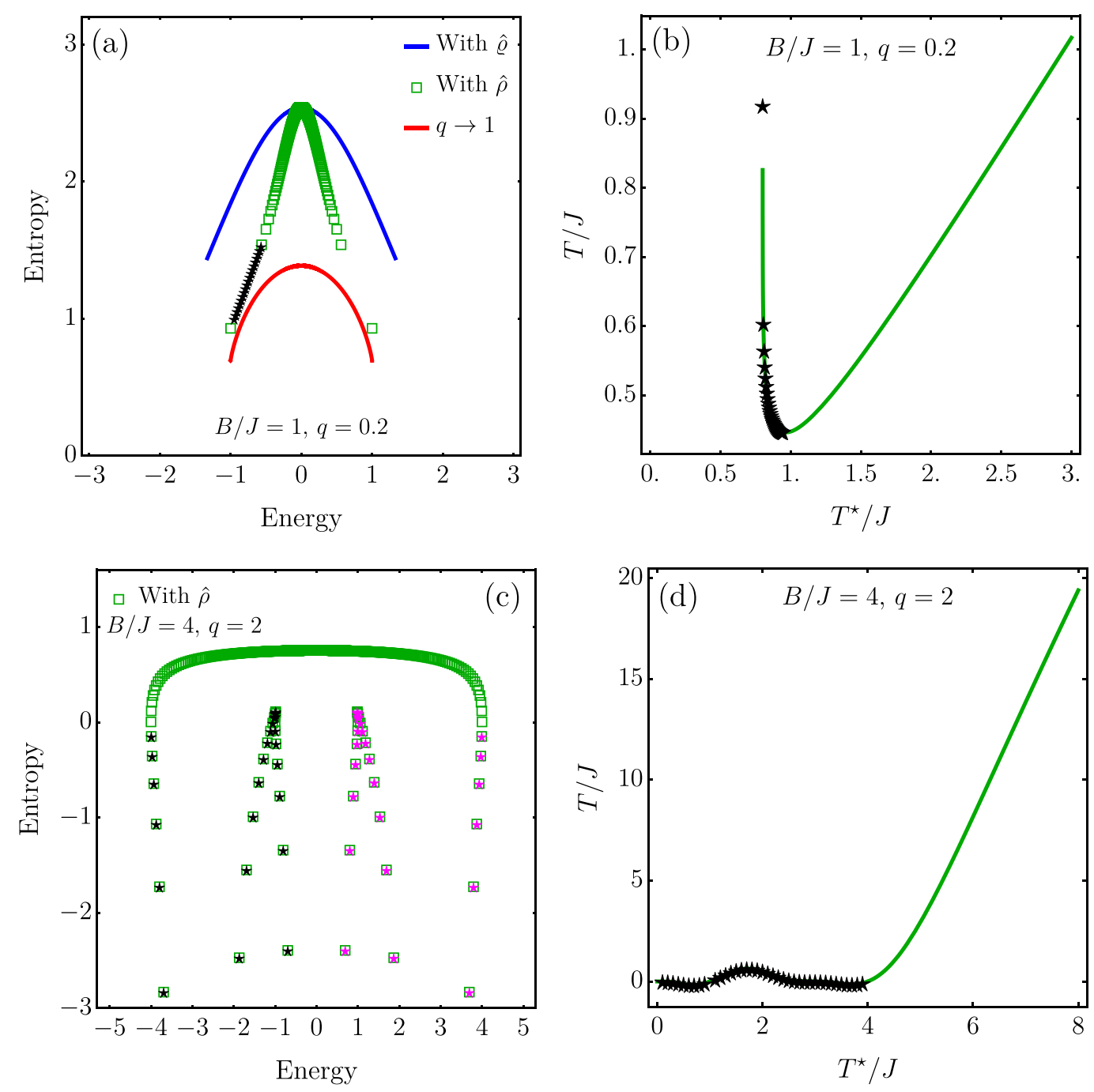

FIG. 3. (a) Entropy as a function of the internal energy, for the non-extensive formalism of Eqs. (6) and (7) (green squares), compared with the formalism related to $\varrho$ (blue line) and the GB case $q \rightarrow 1$ (red line) for $B / J=1$ and $q=0.2$. The black stars are non-physical (positive) temperatures provided by parametrization of Eq. (15), where the thermal observables are not functions of $T$. (b) Identification of the non-physical values of $T$ by using panel (a). In panel (c) $S$ vs. $U$ is computed from $\hat{\rho}$ for $B / J=4$ and $q=2$. The black (pink) stars are the non-physical positive (negative) parametrized temperatures which are identified in panel (d). 
On the other hand, Eq. (16a) is not necessary given that $S$ is already defined in Eq. (6).

The above analysis modifies Fig. 3, but the prescription to eliminate nonphysical temperatures is the same: We discard points where $S(U)$ and $\partial S / \partial U=1 / T$ are not well-behaved or real single-valued functions. With this in mind, the modifications of Figs. 3(a) and 3(c) [Figs. 3(b) and 3(d) remain unaltered] are given in the present document. The black (pink) stars are nonphysical positive (negative) temperatures so that in Fig. 3(a) they correspond to points where $U$ and $S$ are complex functions, and in Fig. 3(c) $S$ is not a single-valued function of $U$.

The named corrections do not affect the main results of the paper, namely, the behavior of the concurrence. Hence, our conclusions remain valid. 